\title{
Morbidity and mortality of patients with endovascularly treated intracerebral aneurysms: does physician specialty matter?
}

\author{
Vernard S. Fennell, MD, Nikolay L. Martirosyan, MD, Sheri K. Palejwala, MD, \\ G. Michael Lemole Jr., MD, and Travis M. Dumont, MD \\ Division of Neurosurgery, Department of Surgery, University of Arizona Medical Center, University of Arizona College of \\ Medicine, Tucson, Arizona
}

OBJECTIVE Endovascular treatment of cerebrovascular pathology, particularly aneurysms, is becoming more prevalent. There is a wide variety in clinical background and training of physicians who treat cerebrovascular pathology through endovascular means. The impact of clinical training background on patient outcomes is not well documented.

METHODS The authors conducted a retrospective analysis of a large national database, the University HealthSystem Consortium, that was queried in the years 2009-2013. Cases of both unruptured cerebral aneurysms and subarachnoid hemorrhage treated by endovascular obliteration were studied. Outcome measures of morbidity and mortality were evaluated according to the specialty of the treating physician.

RESULTS Elective embolization of an unruptured aneurysm was the procedure code and primary diagnosis, respectively, for 12,400 cases. Patients with at least 1 complication were reported in 799 cases (6.4\%). Deaths were reported in 193 cases (1.6\%). Complications and deaths were varied by specialty; the highest incidence of complications $(11.1 \%)$ and deaths (3.0\%) were reported by neurologists. The fewest complications were reported by neurosurgeons $(5.4 \%$; $1.4 \%$ deaths), with a higher incidence of complications reported in cases performed by neurologists ( $p<0.0001$ for both complications and deaths) and to a lesser degree interventional radiologists ( $p=0.0093$ for complications). Subarachnoid hemorrhage was the primary diagnosis and procedure for 8197 cases. At least 1 complication was reported in 2385 cases $(29 \%)$ and deaths in 983 cases $(12 \%)$. The number of complications and deaths varied among specialties. The highest incidence of complications (34\%) and deaths (13.5\%) in subarachnoid hemorrhage was in cases performed by neurologists. The fewest complications were in cases by neurosurgeons $(27 \%)$, with a higher incidence of complications in cases performed by neurologists $(34 \%, p<0.0001)$, and a trend of increased complications with interventional radiologists $(30 \%, p<0.0676)$. The lowest incidence of mortality was in cases performed by neurosurgeons $(11.5 \%)$, with a significantly higher incidence of mortality in cases performed by neurologists $(13.5 \%, p=0.0372)$. Mortality rates did not reach statistical significance with respect to interventional radiologists $(12.1 \%, p=0.4884)$.

CONCLUSIONS Physicians of varied training types and backgrounds use endovascular treatment of ruptured and unruptured intracerebral aneurysms. In this study there was a statistically significant finding that neurosurgically trained physicians may demonstrate improved outcomes with respect to endovascular treatment of unruptured aneurysms in this cohort. This finding warrants further investigation.

http://thejns.org/doi/abs/10.3171/2014.11.JNS141030

KEY WORDS endovascular; morbidity; mortality; cerebral; aneurysm; subarachnoid hemorrhage; coil embolization; vascular disorders

$\mathrm{T}$ HE treatment of intracerebral aneurysms has evolved dramatically with the advent and growth of intracra-

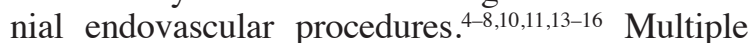
studies have explored the relative mortality and morbidity rates associated with open microvascular treatment as compared with endovascular treatment. ${ }^{10-12,16}$ For patients undergoing primary endovascular treatment, the availability of outcome data by physician specialty is limited. Currently, physicians with training as neurologists, neurosurgeons, and interventional radiologists all use endovas-

ABBREVIATIONS ACGME = Accreditation Council for Graduate Medical Education; ICD-9 = International Classification of Diseases, Ninth Revision; UHC = University HealthSystem Consortium.

ACCOMPANYING EDITORIALS See pp 7-12. DOI: 10.3171/2015.1.JNS142794; DOI: 10.3171/2015.1.JNS142795

SUBMITTED May 6, 2014. ACCEPTED November 14, 2014.

INCLUDE WHEN CITING Published online August 14, 2015; DOI: 10.3171/2014.11.JNS141030. 
cular techniques to treat patients with intracerebral aneurysms. ${ }^{3,6,9,16}$ Even though the endovascular techniques that these 3 types of specialists use are either identical or very similar, the pre-fellowship clinical training that each receives is quite dissimilar. This difference in training could potentially contribute to differences in patient care ideology and subsequent outcomes.

In this retrospective study, we aimed to garner a better understanding of the possible impact of physician specialty on patient outcomes after endovascular treatment. Using a large national database, we compared the complication and mortality rates for patients with unruptured intracerebral aneurysms treated electively, as well as ruptured intracerebral aneurysms presenting with subarachnoid hemorrhage, treated by 3 different types of neurointerventional specialists: neurologists, neurosurgeons, and interventional radiologists.

\section{Methods}

We used the University HealthSystem Consortium (UHC) database, focusing on a 4-year period (October 2009 through September 2013; Q4 2009 to Q3 2013). We noted the records of patients whose unruptured intracerebral aneurysms (primary diagnosis code 437.3, according to the WHO's International Classification of Diseases, Ninth Revision [ICD-9]) were treated with endovascular obliteration (primary procedure, ICD-9 code 39.72 ) and whose ruptured intracerebral aneurysms presented with subarachnoid hemorrhage (primary diagnosis, ICD-9 code 430) and were treated with endovascular obliteration (primary procedure, ICD-9 code 39.72 ).

Then, according to physician specialty, we evaluated patients' morbidity (specifically, the complication rate) and mortality. For 3 types of specialists (neurologists, neurosurgeons, and interventional radiologists), we compared outcomes based on treating physician specialty. The percentages of procedures performed by each specialty group were compared between calendar years. To compare the complication and mortality rates in our study groups, we used a 3 -way chi-square test. For statistical computations, we used GraphPad Prism (version 6, GraphPad Software, Inc.).

\section{Results}

\section{Unruptured Intracerebral Aneurysms}

Elective embolization of unruptured intracerebral aneurysms was the primary procedure and diagnosis, respectively, for 12,400 patients; 799 (6.4\%) had at least 1 complication, and $193(1.6 \%)$ died. The patient complication and mortality rates varied by the specialty of their primary treating physician: neurologists had the highest complication rate $(11.1 \%)$ and the highest mortality rate $(3.0 \%)$. Neurosurgeons had the lowest complication rate $(5.4 \%)$ and a lower mortality rate (1.4\%). As compared with neurosurgeons, neurologists had a significantly higher complication rate $\left(\mathrm{p}<0.0001 ; \chi^{2}=64.4\right)$ as did interventional radiologists $(6.6 \%)$, albeit to a lesser degree $\left(\mathrm{p}=0.0093 ; \chi^{2}\right.$ $=6)$. Neurosurgeons $(1.4 \%)$ and interventional radiologists $(1.3 \%)$ had comparable mortality rates, which were signifi-

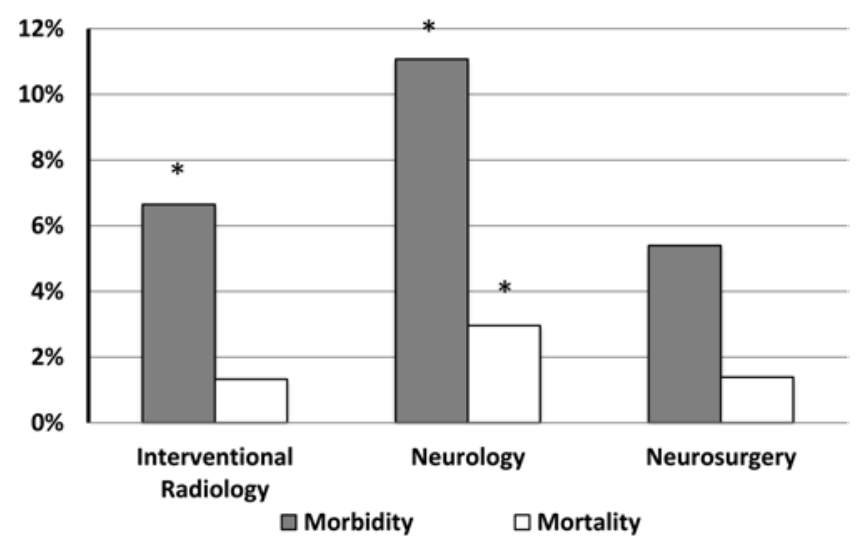

FIG. 1. Bar graph illustrating morbidity and mortality rates of endovascular treatment of unruptured intracerebral aneurysms according to physician specialty. Asterisk indicates significant differences in complication rates $(p<0.05)$.

cantly less than that of neurologists $\left(3.0 \%, \mathrm{p}<0.0001, \chi^{2}=\right.$ 17.2). Complications and deaths of patients with embolization of unruptured intracerebral aneurysms based on treating physician specialty are summarized in Fig. 1.

\section{Subarachnoid Hemorrhage}

Endovascular obliteration of ruptured intracerebral aneurysms with subarachnoid hemorrhage was the primary procedure and diagnosis, respectively, for 8197 patients; $2385(29 \%)$ had at least 1 complication, and $983(12 \%)$ died. Again, the patient complication and mortality rates varied by the specialty of their physician: neurologists had the highest complication rate (34\%) and the highest mortality rate $(13.5 \%)$. Neurosurgeons had the lowest complication rate $(27 \%)$ and the lowest mortality rate $(11.5 \%)$. As compared with neurosurgeons, neurologists had a higher complication rate $\left(\mathrm{p}<0.0001 ; \chi^{2}=21.6\right)$, as did neuroradiologists, although this finding was not statistically significant $\left(p<0.0676 ; \chi^{2}=3.3\right)$. The lowest incidence of mortality was noted in cases performed by neurosurgeons (11.5\%), with a significantly higher incidence of mortality in cases performed by neurologists $(13.5 \%, \mathrm{p}=0.0372$, $\left.\chi^{2}=4.34\right)$. Incidence of mortality was similar to that of neurosurgeons in patients treated by radiologists $(12.1 \%$, $\mathrm{p}=0.4884, \chi^{2}=0.48$ ). Complications and deaths of patients treated using embolization of ruptured intracerebral aneurysms based on treating physician specialty are summarized in Fig. 2. Morbidity and mortality outcomes for unruptured and ruptured aneurysms for all specialties are summarized in Table 1.

\section{Treatment Volume}

A temporal trend suggests an increased volume of aneurysms are being treated by neurologists: the percentage of patients treated by neurologists in the UHC database increased by year (14\% in 2009, $15 \%$ in $2010,18 \%$ in $2011,19 \%$ in 2012, and $21 \%$ in 2013), while there was a decreased overall percentage of patients treated by neurosurgeons $(59 \%$ in $2009,59 \%$ in $2010,56 \%$ in $2011,55 \%$ in 2012, and 51\% in 2013). This trend is particularly pro- 


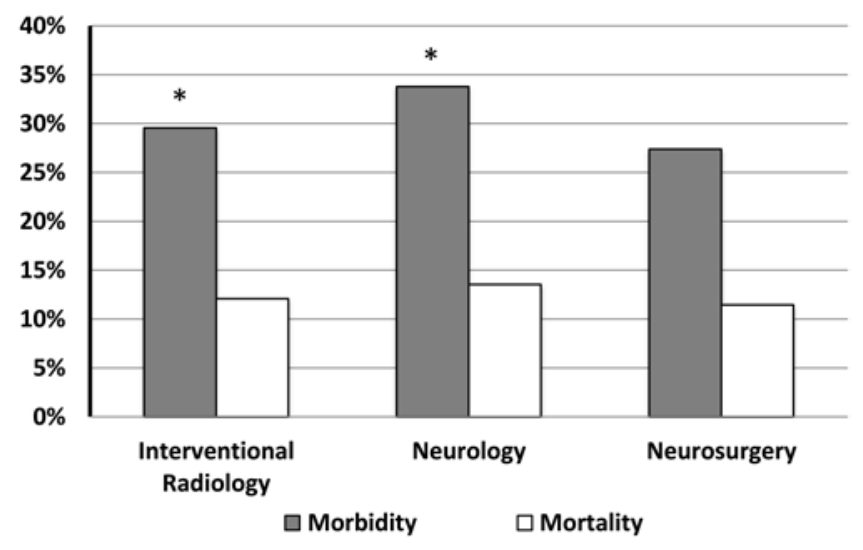

FIG. 2. Bar graph illustrating morbidity and mortality rates of endovascular treatment of subarachnoid hemorrhage according to physician specialty. Asterisk indicates significant differences in complication rates $(p<0.05)$.

nounced for patients with treated unruptured aneurysms. However, the overall number of patients treated by interventional radiologists was relatively constant. This trend is summarized in Fig. 3 upper (for unruptured aneurysms) and Fig. 3 lower (for ruptured aneurysms).

\section{Discussion}

Notable differences in outcomes were found in the 3 different physician specialty groups with respect to the endovascular treatment of aneurysms. For patients with ruptured intracerebral aneurysms and subarachnoid hemorrhages, neurologists had a complication rate of $34 \%$ and a mortality rate of $13.5 \%$, whereas neurosurgeons had the lowest complication rate $(27 \%)$ and the lowest mortality rate $(11.5 \%)$. For patients with unruptured intracerebral aneurysms, a difference in outcomes between specialty designation was also noted: neurologists had a complication rate of $11.1 \%$ and a mortality rate of $3.0 \%$, whereas neurosurgeons had the lowest complication rate $(5.4 \%)$ and a lower mortality rate $(1.4 \%)$. When compared with neurosurgeons, interventional radiologists only trended toward a slightly higher complication rate $(6.6 \%)$ and comparable mortality rate $(1.3 \%)$.

These findings, however, are tempered by the limitations of the UHC database. The UHC includes academic health systems from across the US, with a focus on quality, safety, and excellence (http://www.uhc.edu). The consortium consists of 120 academic medical centers with more than 300 of their affiliated hospitals, who self-report data to the UHC. Individual patient characteristics are not readily available with the current reporting structure. Given the limitations of the UHC database, it is impossible to know whether patients' complications occurred preoperatively, intraoperatively, or postoperatively. Also, we were unable to compare preoperative morbidity of patients in our 3 physician specialty groups; the presence or absence of preoperative morbidity could represent an impactful confounding factor. We are also not able to directly compare individual centers with respect to outcome and volume or specific aneurysm morphology. Without
TABLE 1. Morbidity and mortality rates in unruptured intracerebral aneurysms and subarachnoid hemorrhage treated by elective embolization, according to physician specialty

\begin{tabular}{lcccc}
\hline & \multicolumn{2}{c}{$\begin{array}{c}\text { Unruptured } \\
\text { Intracerebral } \\
\text { Aneurysms (\%) }\end{array}$} & \multicolumn{2}{c}{$\begin{array}{c}\text { Subarachnoid } \\
\text { Hemorrhage (\%) }\end{array}$} \\
\cline { 2 - 5 } Physician Specialty & Morbidity & Mortality & Morbidity & Mortality \\
\hline Neurosurgery & 5.4 & 1.4 & 27 & 11.5 \\
\hline Neurology & 11.1 & 3.0 & 34 & 13.5 \\
\hline Interventional Radiology & 6.6 & 1.3 & 30 & 12.1 \\
\hline
\end{tabular}

patient-matched demographics, our data remain far from complete; nonetheless, we believe that the statistically significant differences by physician specialty that were noted warrant closer inspection and potentially merit prospective evaluation. It should also be noted that there could be significant differences in patient profiles, and subsequently a potentially disparate impact with respect to patient selection. The differences noted could also be attributed to the overall cerebrovascular volume of the treating center as well as the individual treating physician. Hoh et al. previously described the in-hospital morbidity and mortality in the endovascular treatment of intracerebral aneurysms as they pertain to physician and hospital volume. ${ }^{7}$ They noted hospitals with higher volumes ( $>23$ admissions) had better outcomes than those hospitals with lower volumes
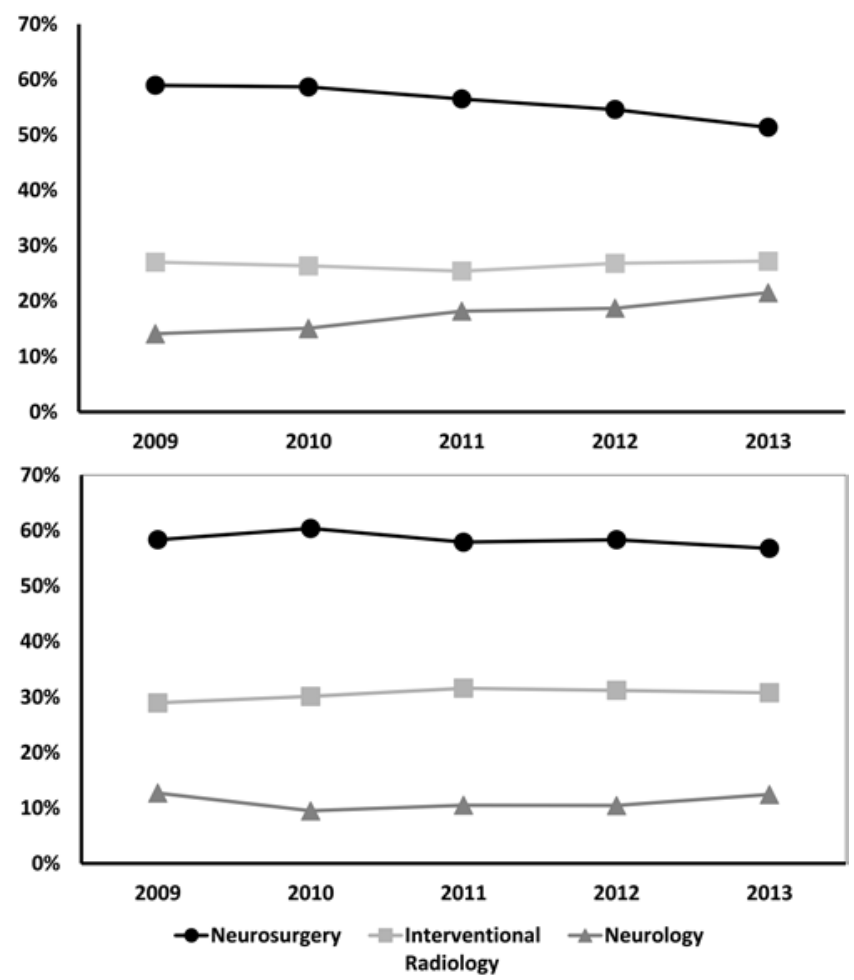

FIG. 3. Graphs illustrating the total percentage of treated patients with unruptured intracerebral aneurysms (upper) and ruptured aneurysms and subarachnoid hemorrhage (lower), according to physician specialty and year. 
( $<4$ admissions). ${ }^{7}$ A similar effect was noted with higher physician volume as well. These investigators did not, however, study any differences that might exist among physician specialty training. To the best of our knowledge, no study currently exists that examines these differences, and therefore our study represents the first of its kind.

We do hypothesize that a lower incidence of complications in patients with intracerebral aneurysms treated by neurosurgeons could potentially be correlated with pre-fellowship training. Specifically, a lower complication incidence in patients treated by neurosurgeons may be a result of a more robust understanding of neurocritical care concepts in the setting of intracerebral aneurysm management. This includes the capability to perform potentially disease-altering procedures when necessary, such as ventriculostomy or craniectomy, with relatively seamless integration. Neurosurgical residents, throughout their typical residency training of 6 or 7 years, frequently encounter critically ill patients with ruptured and unruptured intracerebral aneurysms, must fulfill multiple requirements for not only neurocritical care but also neurocritical cerebrovascular aneurysm care, and must complete an array of microsurgical and endovascular cerebrovascular requirements. ${ }^{1}$ In addition, neurosurgical residents undergo rigorous training in the full spectrum of neurocritical care that necessitates a detailed understanding of acute traumatic neurological injuries, all manner of cerebrovascular emergencies, intracranial tumors, management of intracranial pressure, and bedside intracranial monitoring procedures. ${ }^{1}$ We posit, as a result, there may be differences in total training case exposure.

In contrast, radiology residents, during their typical residency training of 4 or 5 years, may not have that particular clinical exposure; neurology residents, during their typical residency training of 4 years, have limited Accreditation Council for Graduate Medical Education (ACGME) requirements for neurocritical care..$^{2,8,9}$ This difference in residency training requirements may constitute a variable exposure to this particular patient population during a 4-year neurology or 5-year radiology residency. ${ }^{2}$ The heterogeneity of a 1- or 2-year neurointerventional fellowship may be insufficient to make neurointerventionalists equivalent in regard to exposure and understanding concepts of neurocritical care management of patients with intracerebral aneurysms and subarachnoid hemorrhage. Furthermore, not all fellowships with training directed at endovascular treatment of aneurysms are equivalent in their structure or relative requirements.

The issue of standardization of fellowship training is longstanding but remains unresolved. The present 2-year model of neuroendovascular surgery/interventional neuroradiology training developed in 2000 by senior committee members from the American Society of Interventional and Therapeutic Neuroradiology (ASITN), the Joint Section of the American Association of Neurological Surgeons and Congress of Neurological Surgeons (AANS/CNS), and the American Society of Neuroradiology (ASNR) may be insufficient to ensure adequate clinical and technical skills for physicians with different training backgrounds. ${ }^{6}$ In this model, a program lasts 2 years: the first year is spent on preparatory skills, and only the second year is devoted to advanced clinical and technical training. Such a program builds on a foundation of a completed neurosurgical, neurological, or radiological residency followed by a diagnostic neuroradiology fellowship..$^{10}$ However, for some physicians without significant prior experience, 2 years may not be adequate exposure to neurocritical care concepts in regard to aneurysm care.

Complicating matters, there has been a call for recognition of formal integration of neuroendovascular skills into neurosurgical residency and is now part of the current case requirements. ${ }^{1,3,5}$ And concerns have been raised about training an excessive number of neurointerventionalists. ${ }^{4}$ The already immense number of neuroendovascular practitioners has moved more neurointerventionalists into community settings, thereby decentralizing centers of excellence. ${ }^{4}$ A greater influx of neurology-trained physicians treating intracerebral aneurysms is potentially reflected in the steady increase of patients treated by neurologists that we observed in our study, a possible explanation of the differences we found with respect to patients' morbidity and mortality.

With so many neurointerventionalists now practicing, and coming from such different physician specialties, this question remains: what is the way to standardize training to optimize patient outcomes? The ACGME guidelines, although relatively recent, are already outdated in the face of this rapidly evolving field. In the modern age of surgeons with dual training in microsurgical clip ligation and endovascular coil embolization of intracerebral aneurysms, the multiphysician model of surgeon, interventionalist, and intensivist to treat patients with cerebral aneurysms may represent an unsafe anachronism. ${ }^{9}$ The current model may disproportionately affect the outcomes of physicians treating aneurysms at lower volume centers and could significantly alter the overall outcomes that are reported here.

Furthermore, we believe that it is time to shift the focus toward patient outcomes; the resulting data (such as from studies like this) should be used to fine-tune the fellowship training process and standardize the prerequisite residency training. Regardless of the length or shape of training obtained by physicians treating aneurysms with endovascular techniques, patient care may ultimately benefit from an intensive focus on critical care management of patients and potentially lifesaving surgical procedures, such as ventriculostomy in addition to catheter-based skills as part of advanced post-residency fellowship training.

\section{Conclusions}

Physicians of varied specialties use endovascular treatment of ruptured and unruptured intracerebral aneurysms. In this study we found that patients treated by neurosurgeons, when compared with neurologists and to a lesser degree with interventional radiologists, had improved outcomes. Our finding of lower complication and mortality rates for the patients treated by neurosurgeons is compelling and warrants further investigation.

\section{Acknowledgment}

The authors would like to thank Mary Knatterud for assistance in preparing this manuscript. 


\section{References}

1. Accreditation Council for Graduate Medical Education: ACGME Program Requirements for Graduate Medical Education in Neurological Surgery. (https:// www.acgme.org/acgmeweb/Portals/0/PFAssets/ ProgramRequirements/160_neurological_surgery_2016.pdf) [Accessed May 27, 2015]

2. Accreditation Council for Graduate Medical Education: ACGME Program Requirements for Graduate Medical Education in Neurology. (http://www.acgme.org/ acgmeweb/portals/0/pfassets/programrequirements/180_ neurology_07012014.pdf) [Accessed May 27, 2015]

3. Chaturvedi S, Dumont AS: The learning curve for neuroendovascular procedures: how important is it? Neurology 72:1974-1975, 2009

4. Chaudhary N, Gemmete JJ, Thompson BG, Xi G, Pandey AS: Iron-potential therapeutic target in hemorrhagic stroke. World Neurosurg 79:7-9, 2013

5. Harbaugh RE, Agarwal A: Training residents in endovascular neurosurgery: Neurosurgery 59 (5 Suppl 3):S277-S281, S3-S13, 2006

6. Higashida RT, Hopkins LN, Berenstein A, Halbach VV, Kerber C: Program requirements for residency/fellowship education in neuroendovascular surgery/interventional neuroradiology: a special report on graduate medical education. AJNR Am J Neuroradiol 21:1153-1159, 2000

7. Hoh BL, Rabinov JD, Pryor JC, Carter BS, Barker FG II: In-hospital morbidity and mortality after endovascular treatment of unruptured intracranial aneurysms in the United States, 1996-2000: effect of hospital and physician volume. AJNR Am J Neuroradiol 24:1409-1420, 2003

8. Hopkins LN, Higashida RT, Piepgras DG: Perspectives on training standards in neuroendovascular therapeutics. Neurosurg Clin N Am 11:187-190, x, 2000

9. Hopkins LN, Strother CM: Neuroendovascular training. Neurosurg Clin N Am 5:565-567, 1994

10. Lanzino G, Murad MH, d'Urso PI, Rabinstein AA: Coil embolization versus clipping for ruptured intracranial aneurysms: a meta-analysis of prospective controlled published studies. AJNR Am J Neuroradiol 34:1764-1768, 2013

11. Lawson MF, Neal DW, Mocco J, Hoh BL: Rationale for treating unruptured intracranial aneurysms: actuarial analysis of natural history risk versus treatment risk for coiling or clipping based on 14,050 patients in the Nationwide Inpatient Sample database. World Neurosurg 79:472-478, 2013
12. Meyers PM, Schumacher HC, Higashida RT, Derdeyn CP, Nesbit GM, Sacks D, et al: Reporting standards for endovascular repair of saccular intracranial cerebral aneurysms. AJNR Am J Neuroradiol 31:E12-E24, 2010

13. Miyachi S, Okamoto T, Kobayashi N, Kojima T, Hattori KI, Nakai K, et al: Nagoya University training system for neuroendovascular therapists. Interv Neuroradiol 10 (Suppl 1):103-106, 2004

14. Qureshi AI, Janardhan V, Memon MZ, Suri MFK, Shah QA, Miley JT, et al: Initial experience in establishing an academic neuroendovascular service: program building, procedural types, and outcomes. J Neuroimaging 19:72-79, 2009

15. Richling B: The way to an open and structured training for neuroendovascular therapy in Europe: what is the current situation? World Neurosurg 77:259-260, 2012

16. Wiebers DO, Whisnant JP, Huston J III, Meissner I, Brown RD Jr, Piepgras DG, et al: Unruptured intracranial aneurysms: natural history, clinical outcome, and risks of surgical and endovascular treatment. Lancet 362:103-110, 2003

\section{Disclosure}

The authors report no conflict of interest concerning the materials or methods used in this study or the findings specified in this paper.

\section{Author Contributions}

Conception and design: Dumont. Acquisition of data: Dumont, Fennell, Palejwala. Analysis and interpretation of data: Dumont, Fennell, Martirosyan, Palejwala. Drafting the article: Fennell, Martirosyan, Palejwala. Critically revising the article: Dumont, Martirosyan, Lemole. Reviewed submitted version of manuscript: Dumont, Fennell, Martirosyan, Lemole. Approved the final version of the manuscript on behalf of all authors: Dumont. Statistical analysis: Dumont, Fennell, Martirosyan. Administrative/ technical/material support: Lemole. Study supervision: Dumont, Lemole.

\section{Correspondence}

Travis M. Dumont, Neurovascular Surgery Program, University of Arizona Medical Center, University of Arizona, $1501 \mathrm{~N}$. Campbell Ave., Rm. 4303, Tucson, AZ 85724-5070. email: tdumont@surgery.arizona.edu. 